\title{
Ectopic breast localization wire in the pleural cavity: A case report
}

\author{
XUELU LI ${ }^{1 *}$, DANDAN ZHU ${ }^{2 *}$, MAN LI $^{1}$ and ZUOWEI ZHAO ${ }^{1}$ \\ Departments of ${ }^{1}$ Breast Surgery and Oncology, and ${ }^{2}$ Critical Care Medicine, \\ The Second Hospital of Dalian Medical University, Dalian, Liaoning 116027, P.R. China
}

Received July 24, 2017; Accepted September 11, 2017

DOI: $10.3892 / \mathrm{mco} .2018 .1593$

\begin{abstract}
Ultrasound is an easily accessible and cost-effective imaging method that is not associated with radiation exposure. Ultrasound-guided wire localization is a reliable method for resecting non-palpable breast lesions. Preoperative ultrasound-guided wire localization has the advantage of a shorter operative time, and the breast lesions may be accurately excised with less tissue injury. We herein report an unusual case of an ectopic breast localization wire in the pleural cavity in a 47-year-old female patient with a history of right-sided breast cancer, who received left segmental mastectomy due to a newly identified left-sided breast lesion. The wire was identified with intraoperative X-ray and computed tomography imaging and was successfully removed under thoracoscopic guidance. The patient recovered uneventfully. To the best of our knowledge, this is the first report on localization wire-related complications in the English literature.
\end{abstract}

\section{Case report}

A 47-year-old woman with a 1-year history of resected (right modified radical mastectomy) invasive breast carcinoma of the right breast (pT1N0M0) presented to the Department of Breast Surgery of The Second Hospital of Dalian Medical University (Dalian, China) for a regular follow-up visit in January 2014. Breast ultrasound examination revealed a neoplasm in the left breast, with sand-like calcifications $\left(0.6 \times 0.7 \mathrm{~cm}^{2}\right.$, BI-RADS score IVB). Due to the previous history of a malignant tumor, further surgery was required to reach an accurate diagnosis. Therefore, segmental left mastectomy was scheduled to remove the lesion. Ultrasound-guided wire localization of the non-palpable left breast lesion was performed preoperatively at the Department of Ultrasound. Due to the numerous surgeries

Correspondence to: Dr Zuowei Zhao or Dr Man Li, Department of Breast Surgery and Oncology, The Second Hospital of Dalian Medical University, Dalian, Liaoning 116027, P.R. China

E-mail: dmuzhaozuowei@163.com

E-mail:dmuliman@163.com

${ }^{*}$ Contributed equally

Key words: wire localization, ultrasonography, breast cancer scheduled on that day, the patient was required to wait for $\sim 6 \mathrm{~h}$. When the patient was finally taken to the operating theater, the localization wire had disappeared. It was first considered that the localization wire had become detached and fallen off; however, we were unable to find it. We then considered the possibility that the wire was located within the mammary tissue, and proceeded with the segmental mastectomy, along with the preoperative skin marker; however, following tumor resection, the localization wire could not be identified in the mammary gland. The intraoperative pathology report revealed a benign breast lesion (breast fibroadenoma). As the possibility that the localization wire had entered the pleural cavity could not be excluded, plain chest X-ray was performed intraoperatively (Fig. 1), followed by a computed tomography (CT) scan to accurately evaluate the location of the wire. The wire was located in the left pleural cavity (Fig. 2) and it was removed by the thoracic surgeons under thoracoscopic guidance. After 1 week, the patient was re-examined with a CT scan and was released from the hospital.

\section{Discussion}

Preoperative ultrasound-guided wire localization is an effective method for assisting surgeons in resecting non-palpable breast lesions (1-4). However, little is known on the complications associated with the use of ultrasound-guided wire localization. We herein present an unusual case of an ectopic breast localization wire in the pleural cavity. To the best of our knowledge, this is the first report of this type of complication.

Apart from ultrasound-guided wire localization, alternative methods for breast tumor localization include preoperative skin markers and intraoperative ultrasound. However, these techniques may result in inaccurate tumor localization due to skin mobility and body position changes (3-5). Two main factors may have contributed to this unusual complication: One was the unusually long waiting period, during which the localization wire may have been pushed out of its original position, which is considered to be quite dangerous. In addition, the depth of the localization wire was questioned. If the localization wire is erroneously inserted to a greater depth, it may enter the pleural cavity. However, the pectoralis major is a thick fan-shaped muscle with a tough fascia. In fact, it would be quite difficult to advance the wire to that depth; furthermore, the ultrasound doctors who performed the procedure are highly experienced ( $>10$ years of clinical practice) and were assisted by experienced surgeons. Therefore, it is extremely unlikely that the localization 


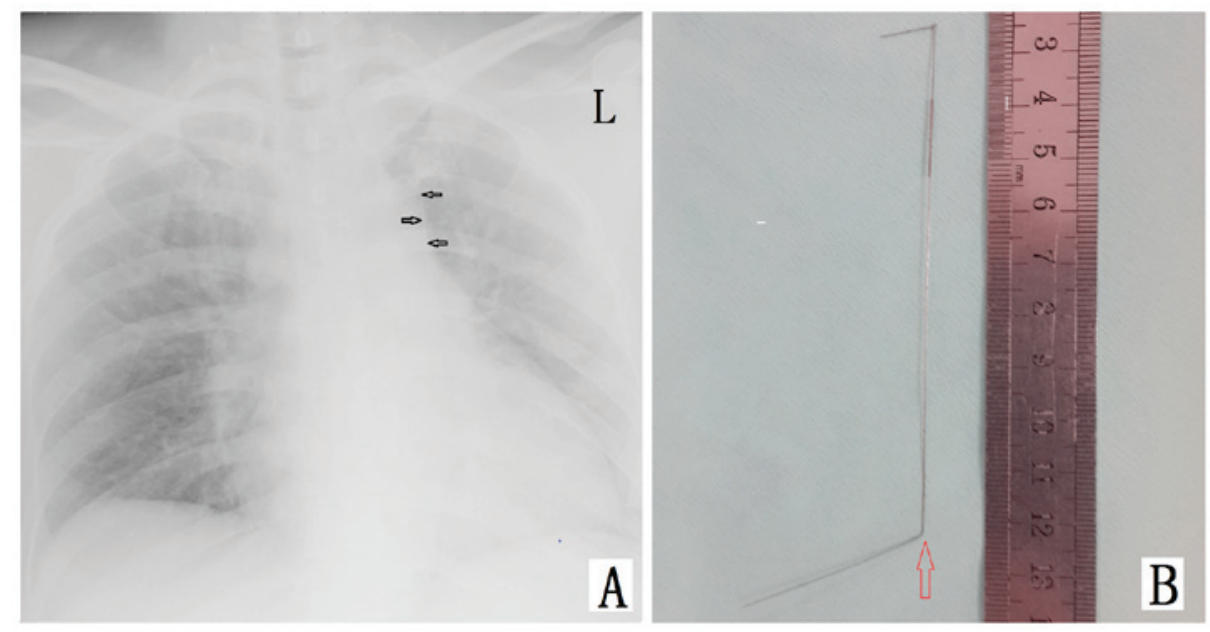

Figure 1. (A) The plain chest X-ray revealed a high-density shadow (arrows). (B) The length of the residual localization wire was $\sim 15 \mathrm{~cm}$. The localization wire had a J-shaped end, and was bended by the thoracoscope instrument during removal (arrow).

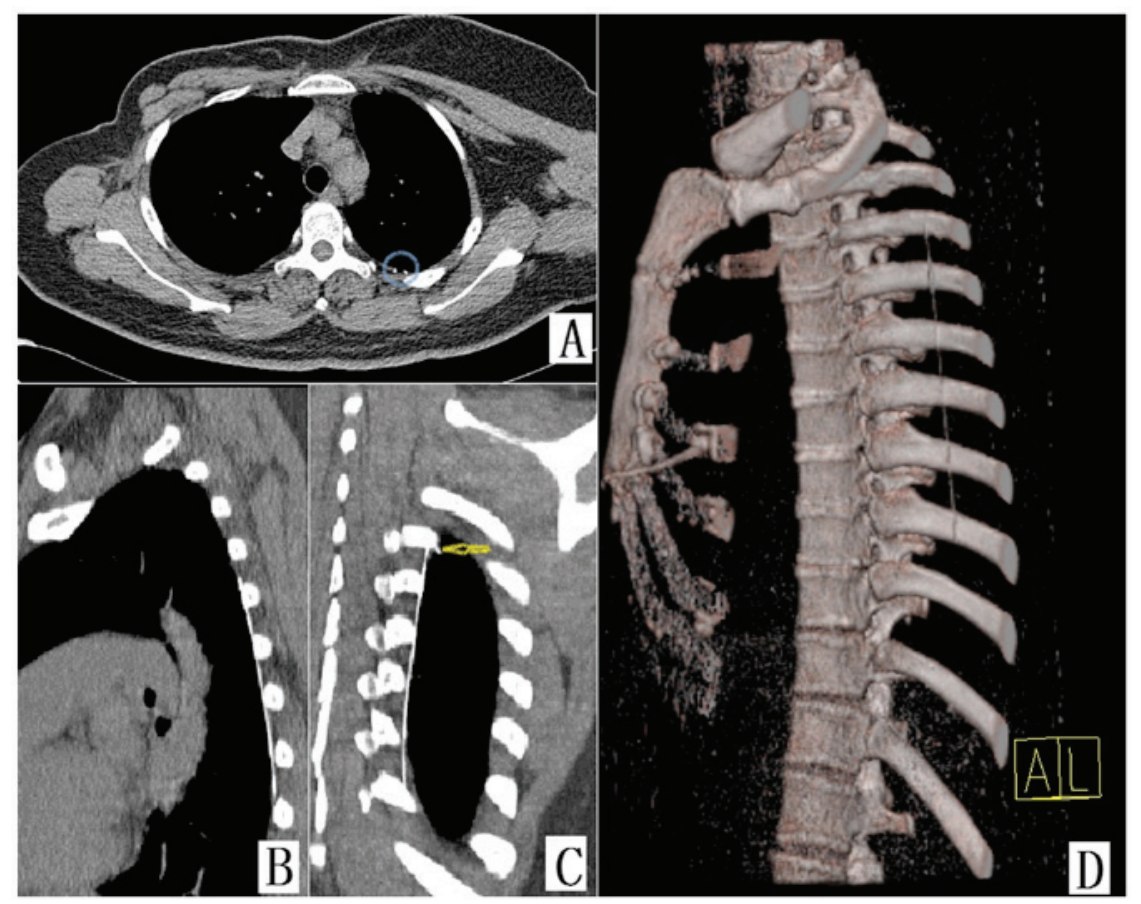

Figure 2. Computed tomography (CT) scan. (A) Two point-like high-density shadows (blue circle) confirmed the location of the J-shaped end; (B) shows the length and (C) the location of the localization wire at different anatomical regions. The J-shaped end is indicated by the yellow arrow. (D) Representative image of the $3 \mathrm{D}$ reconstruction.

wire was introduced into the pleural cavity under ultrasound guidance, without any patient-reported symptoms. None of the two possibilities appears to be a plausible explanation for this complication. However, in the future, the operation time should be better coordinated with the time of ultrasound-guided wire localization to reduce the waiting period.

Needle-track seeding is a rare but important complication of ultrasound-guided procedures (1-5). In the present case, as the lesion was reported to be benign based on postoperative pathology, the risk of pleural metastasis did not appear to be a concern. In addition, the patient had fully recovered after 1 week. However, the risk of needle-track seeding, as well as that of other high-risk complications, such as lethal left pneu- mothorax and severe pulmonary injury, should not be ignored. To the best of our knowledge, there have been no reports of an ectopic breast localization wire in the pleural cavity in the English literature to date.

The patient provided written informed consent to the publication of the case details and associated images, and the study protocol and the sample collection were approved by the Ethics Committee of Dalian Medical University.

\section{Acknowledgements}

The present study was supported by the National Natural Science Foundation of China (grant nos. 81071127, 81471751 
and 81673762 to Dr Zuowei Zhao; grant no. 81650018 to Dr Man Li), and the Provincial Natural Science Foundation of Liaoning (grant no. 2014921059 to Dr Zuowei Zhao; grant no. 2014023025 to Dr Man Li).

\section{Competing interests}

The authors declare that they have no competing interests.

\section{References}

1. Plecha D, Bai S, Patterson H, Thompson C and Shenk R Improving the accuracy of axillary lymph node surgery in breast cancer with ultrasound-guided wire localization of biopsy proven metastatic lymph nodes. Ann Surg Oncol 22: 4241-4246, 2015.
2. Krekel NM, Zonderhuis BM, Stockmann HB, Schreurs WH, van der Veen H, de Lange de Klerk ES, Meijer S and van den Tol MP: A comparison of three methods for nonpalpable breast cancer excision. Eur J Surg Oncol 37: 109-115, 2011.

3. Dua SM, Gray RJ and Keshtgar M: Strategies for localisation of impalpable breast lesions. Breast 20: 246-253, 2011.

4. Postma EL, Verkooijen HM, van Esser S, Hobbelink MG, van der Schelling GP, Koelemij R, Witkamp AJ, Contant C, van Diest PJ, Willems SM, et al: Efficacy of 'radioguided occult lesion localisation' (ROLL) versus 'wire-guided localisation' (WGL) in breast conserving surgery for non-palpable breast cancer: A randomised controlled multicentre trial. Breast Cancer Res Treat 136: 469-478, 2012.

5. Ishizuna K, Ota D, Okamoto J, Fukuuchi A, Tanaka R, Fujii A, Mori M and Nishi T: A case of mucinous carcinoma of the breast in which needle tract seeding was diagnosed by preoperative diagnostic imaging. Breast Cancer 18: 324-327, 2011. 